\title{
DESIGN AND IMPLEMENTATION OF SEAT OCCUPANCY DETECTION SYSTEM
}

\author{
B. Kommey, E.O. Addo., and K. A. Adjei \\ Department of Computer Engineering, KNUST-Kumasi, \\ bkommey.coe@knust.edu.gh \\ erny.addo@gmail.com \\ terry.adjei@gmail.com
}

\begin{abstract}
Location of appropriate seats in seating areas of theaters remains a significant challenge that patrons of these enterprises face. There is therefore, the need for seat occupancy monitoring system to provide readily accessible seat occupancy information to clients and management of these halls. This paper presents the design and implementation of a low cost seat occupancy detection and display system which is capable of monitoring seat occupancy in halls efficiently. The system uses capacitive seat sensors which is designed based on the loading mode technology. It detects the presence of a human occupant using a single electrode. Occupancy data is relayed to a WiFi-enabled microcontroller unit which processes the data and wirelessly transfers the processed data to a central base station over a local area network for graphical and numerical display. Commands are also transferred from the base station to the microcontroller units when needed. Theoretical and empirical results show that the system is able to achieve seat occupancy monitoring accurately, neatly and cost effectively.
\end{abstract}

Keywords: Capacitive sensing, seat occupancy, sensor cluster, microstrip transmission line, Wi-Fi

\section{INTRODUCTION}

Patrons of theaters often encounter the difficulty of locating appropriate seats for viewing of shows. In large capacity halls, the above problem is worsened as new patrons stand for longer periods, scanning the entire hall in order to locate available seats. Additionally, after empty seats are spotted, seated, clients go through the repetitive activity of questioning of nearby seated occupants whether the empty seats are al- ready taken by an absent occupant or not. Families or other groups of related clients who patronize these shows encounter more of these problems in their search of fitting seat sets with preferred locations. Many hall establishments therefore engage the service of ushers to resolve the above listed problems which is expensive because these attendants are often needed only few times during a show. These personnel also engage in distracting activities such as 


\section{Kommey et al.}

noisy chatting, waving hands to beckon to clients and getting in the way of customers.

Lastly, ushers are often tasked to determine this attendance by undertaking tiring and errorprone manual counting processes. Since these activities are also distracting, they are often limited in terms of frequency. Real-time seat availability information is therefore not easily and quickly available to newly arrived clients and hall management.

In this paper, the design and implementation of a low cost, occupancy detection and monitoring system is presented. This system which is accurate and efficient uses capacitive sensing technology for human occupancy detection and wireless technology for communication.

\section{RELATED WORKS}

Seat occupancy monitoring involves techniques to sense the presence of a human occupant and relay this information to an appropriate device for decisions to be taken. The essential nature of the information obtained from this activity has prompted a number of studies aimed at developing systems for seat occupancy detection with a number of prototypes developed. Some of these occupancy detection systems have been developed for use in theaters (Blinkilde et al., 1975), (Grunfeld, 2012), intelligent automobile safety device applications (Zeidler et al., 1997), (Zangl et al., 2008), (George et al., 2009), (Satz et al., 2009), and airplane seat location mapping (vonDoenhoff et al, 2008). Gaugler et al. in 1941 worked on a chair for motion picture theaters. The principal object of Gaugler's invention was the provision of signal lights on the top, rear, and front of each seat which automatically lights when the patron rises and leaves the seat and which goes off when the seat is occupied. The multiplicity and positions of lights installed on each seat renders the system, power inefficient and the lighting scheme distracting, especially if many seats are empty. Additionally, the system cannot be easily ported to already-built seats as the circuitry was built into the seats.

The design proposed by Baron et al. (2000) presents a system capable of providing information regarding occupancy on individual seats and a display providing information relating to seat occupancy and the seating area. The system has multi-colored lights mounted on the tops of the seats for indicating occupancy status. The lights were controlled by sensors mounted in the seats and manual switches mounted on the seats. The system was all hardwired for the purposes of power supply, control, and relay of information to the display. This resulted in excessive installation cost, difficulties in troubleshooting faults, and the hall becoming untidy with time when the cables are not well kept. A recent work conducted by Hefferon (2014) involved a wireless reporting device with a pressure sensor component affixed to seats that detects when a patron has sat in the chair. The method employed in determining an occupant's presence is problematic. Since the seat is said to be occupied when an occupant's body weight forces the seat's sitting area to push against the pressure sensor, significantly heavy objects placed on the seat could be mistaken for human occupants.

\section{MATERIALS AND METHODS}

\section{System architecture}

The presented occupancy detection and display system consists of capacitive seat sensors, $\mathrm{WiFi}$ -enabled microcontrollers and a base station as shown in Fig. 1. Each seat sensor uses the loading mode capacitive sensing technique and was connected to a microcontroller through copper wires as illustrated in Fig. 2. Seats and their sensor were clustered and each grouping was assigned one microcontroller which was affixed to one of the seats in the cluster. This microcontroller unit is responsible for processing data received from the seat sensors and relaying the processed data to the base station. An access point was used as an external gateway device for the wireless local area network over which system communication occurs. It routes processed occupancy status data and commands transmitted between the base station and the micro controller units. The base station was connected to a display device upon which the occupancy status of all seats in the auditorium were projected graphically and numerically. 


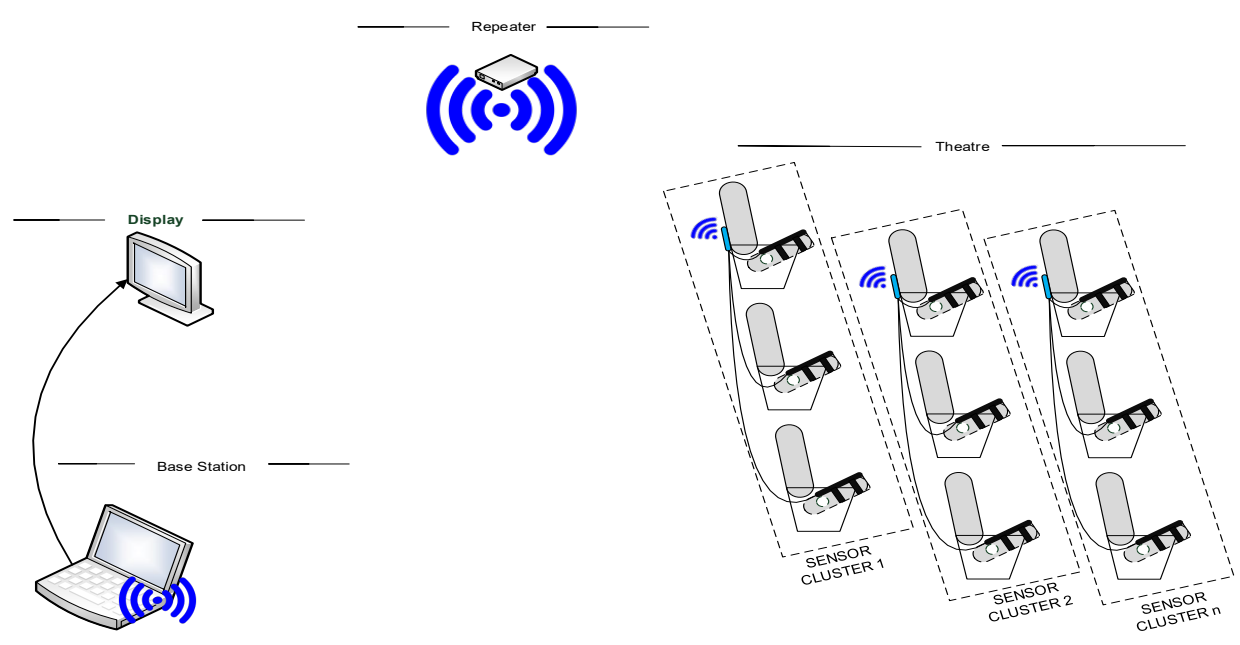

Fig. 1: Architecture of system

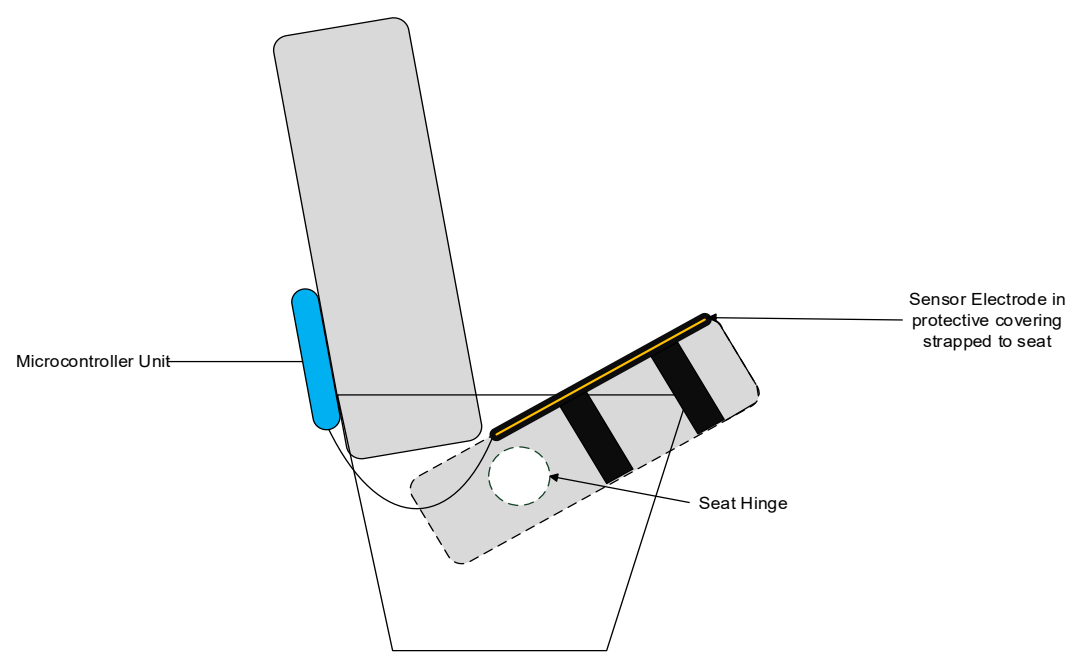

Fig. 2: A seat with sensor and microcontroller unit affixed

\section{System communication}

The system uses minimal wiring for sensor to microcontroller unit connection and applies WiFi technology for communication between the microcontrollers that gather and encode data; and the base station. The quality of wireless communication is heavily dependent on certain important factors. Among these are the physical surroundings in which the transmissions occur and the amount of and the amount 


\section{Kommey et al.}

of electromagnetic interference or noise present. A Wi-Fi network provide wireless connectivity between the sensor cluster microcontrollers and the base station. This enables the sensor units to be added or removed from the system with very little modification to the network. Accurate data transmission is ensured due to intrinsic data-packet building capability of Wi-Fi. The Carrier Sense Multiple Access with Collision Avoidance (CSMA/CA) standard employed in this communication system adds a desirable feature of packet collision avoidance. The topology of the network is a star configuration with the base station at the center. Thus the microcontrollers do not communicate with each other but each with the base station only over the network. The discrete sensor units on the network can also be recognized and monitored independently by the base station.

\section{Capacitive sensor}

The capacitive sensor idea was built on the concept of the basic parallel plate capacitor. Capacitance is defined as the amount of electrical charge stored between two nonconnected conductive plates which changes with the voltage difference between the plates. The capacitance depends primarily on the dielectric substrate, the overlapping area of the plates and the distance between them. The capacitance, $C$ of a parallel plate capacitor shown in fig. 3 is given by

$C=\frac{A \in_{0} \epsilon_{r}}{d}=\frac{\pi r^{2} \in_{0} \epsilon_{r}}{d}$
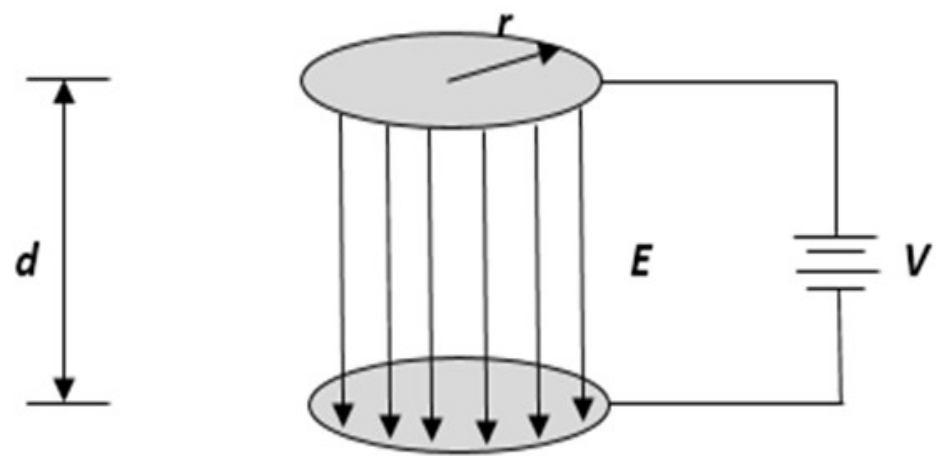

Fig. 3: A basic two parallel plate capacitor

Journal of Science and Technology @ KNUST August 2017 where $\epsilon_{r}$ is the dielectric constant of the separating material and $\epsilon_{0}$ is the permittivity of free

space $\left(8.85 \times 10^{-12} F / m\right) A, d, E$, and $V$ are

the overlapping plate area, distance between plates, induced electric field, and potential difference across plates respectively.

From Equation 1, it was observed that only variations in $\epsilon_{r}$ can affect the capacitance, since $\epsilon_{0}$ is a constant and $A$ and $d$ are assumed to stay constant. As shown in fig. 4, the sensor setup uses uses two of the microcontroller's pins: the stimulus pin $V_{s}$ and the response pin $V_{R}$, which are configured as output and input pins respectively. A $50 \mathrm{M} \Omega$ resistor was connected between the stimulus pin and the sensor electrode. The high value resistor guaranteed sufficient charge and discharge times of the resulting capacitors. The response pin taps the connections between the electrode and the resistor. The electrode is placed in a piece of nonconducting casing made of polyethylene foam and cotton as shown in fig. 5. The casing was meant to protect the sensor connections and make it more comfortable for use. It was secured in place by strapping it to the seat. The electrode is made from a thin circular aluminium foil and the electrode was made circular in shape in order to decrease fringe capacitances that forms around the edges. To accommodate the effect of fringing, the radius of the sensor 
electrode was increased by $13 \%$ for theoretical calculations (Hoch, 1926).

The sensor was built based on the capacitive sensor mathematical model developed by Zeeman et al. (2013) which established a relationship between the capacitance of unoccupied and occupied seat sensors. The auditorium floor and building was used as the ground electrode. The sensor plate with respect to the floor and ceiling 'ground electrodes' with the separating non-conducting areas from the capacitors $C_{l}$ and $C_{2}$ respectively as shown in Fig. 7 . The model derives an effective relative dielectric permittivity by modelling the capacitive sensor as a microstrip transmission line since the dielectric substrate constitutes partly of air. This microstrip transmission line was made up of the sensor electrode of radius $r$ and a wider ground plane, separated by a dielectric of thickness $d$. From Bahl et al. (1977), the effective relative permittivity $\epsilon_{e f f}$ can be estimated

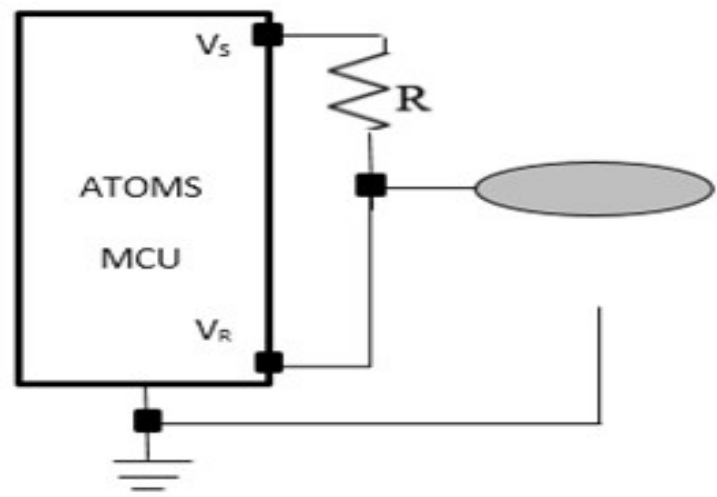

Fig. 4: Capacitive sensor setup

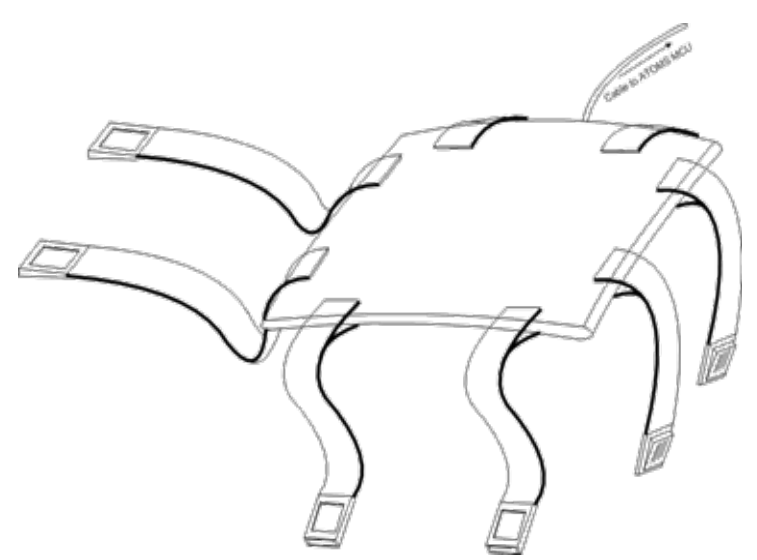

Fig. 5: Protective covering for sensor electrode 


\section{Kommey et al.}

using the following expressions:

when $\left(\frac{2 r}{d}\right)<1$

$\epsilon_{\theta f f}=\frac{\epsilon_{\gamma}+1}{2}+\frac{\epsilon_{r}-1}{2}\left[\left(1+6\left(\frac{d}{r}\right)\right)^{\frac{-1}{2}}+0.04\left(1-2\left(\frac{r}{d}\right)\right)^{2}\right]$

when $\left(\frac{2 r}{d}\right) \geq 1$

$\epsilon_{\text {eff }}=\frac{\epsilon_{r}+1}{2}+\frac{\epsilon_{r}-1}{2}\left(1+6\left(\frac{d}{r}\right)\right)^{\frac{-1}{2}}$

$C_{1}$ remains constant since the position of the chair is fixed. However, $C_{2}$ varies and can either be in two states: unoccupied (UNOCP) or occupied (OCP). The total capacitance in the unoccupied and occupied states will be

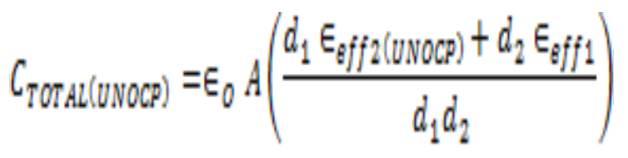

$C_{\text {TOTAL (OCP) }}=\epsilon_{0} A\left(\frac{d_{1} \epsilon_{\theta f f 2(O C P)}+d_{2} \epsilon_{\theta f f 1}}{d_{1} d_{2}}\right)$

where $\epsilon_{\text {eff } 2(U N O C P)}$ and $\epsilon_{\text {eff2(OCP) }}$ are the effective dielectric constants above an unoccupied and occupied seat sensor electrodes respectively. $\epsilon_{\text {eff } 1}$ is the effective dielectric constant below the sensor electrode, $d_{1}$ and $d_{2}$ are the dielectric substrate heights of $C_{1}$ and $C_{2}$ respectively.

The sensor electrode has a radius, $r=0.1 \mathrm{~m}$ and the seat which is made of polyethylene foam has $\epsilon_{r}$ of 2.26 . With $d_{l} \mathrm{~m} 0.115 m, \epsilon_{\text {eff } 1}$ is calculated as 1.86 from equation 2 since

$\left(\frac{2 r}{d 1}<1\right)$ The metals in the seat are respons-

ible for the small $d_{1}$ as it effectively reduces the distance to electrical ground. The protective cover of sensor electrode was made of cotton and rubber foam which have dielectric constant of 1.3 and 3.2 respectively. The cover's $\epsilon_{r}$ is therefore approximated as 2.25 .

For $d_{2}=0.05 m,\left(\frac{2 r}{d 2}>1\right)$ and thus from equation

(3) $\epsilon_{\text {eff } 2(U N O C P)}=1.9375$. The presence of a

human occupant significantly increases the effective dielectric constant, $\epsilon_{\text {eff } 2}$ resulting in a change in capacitive interaction (Fig. 6). Since the human body has $70.4 \%$ (Watson et al., 1980), we can approximate the human body's dielectric properties with that of water that is $\epsilon_{r}=74$ at $37^{\circ} \mathrm{C}$ (Schroeder et al., 2008). The height of the ceiling of the setup room from the sensor electrode $d_{2}$ in the setup was $2.33 m$ and $d_{1}=0.115 m .\left(\frac{2 r}{d 2}<1\right)$ therefore from Equation (2) $\epsilon_{e f f 2}$ is calculated as 41.8.

With $A=\pi(0.1 \times 1.13)^{2}$, the total unoccupied state capacitance, $C_{\text {TOTAL(UNOCP) }}$ is calculated using Equation (4) as $06.37 p F$. Calculating the total occupied state capacitance $C_{\text {TOTAL(OCP) }}$ from Equation (5) with the same $\mathrm{A}$, gives a value of $12.974 p F$. These values are set as thresholds in software for comparison during operation and subsequent occupancy detection.

In measuring the capacitance, the stimulus pin was set to logical high $\left(V_{S}\right)$ and a timer was then started. The stimulus pin charges the capacitor until the response pin reaches the logic high threshold $\left(V_{H T}\right)$. The timer was briefly stopped for the response pin to charge to $V_{c c}$ completely The stimulus pin was set to logic low $\left(V_{L}\right)$ and timer was reactivated. This caused the capacitor to discharge until the response pin reached the logic low threshold $\left(V_{L T}\right)$. The timer was reset and the cycle repeat- 
ed. The timer measured the time taken for the response pin to charge to the opposite logic. The value of the timer is directly proportional to the $R C$ timeconstant. Since $R$ stays constant, the measured time solely depends on the total capacitance, $C_{\text {TOTAL }}$ whose variation is also as a result of changes in capacitance $C_{2}$.

The sensor cluster microcontroller

The sensor clustered microcontrollers have I/O pins to which each sensor leads were connected. These devices were built on the Texas Instruments (TI) CC3200 microcontroller. The TI CC3200 microcontroller is equipped with a $\mathrm{Wi}$ Fi network processor and therefore made the device Wi-Fi capable. Figs. 9, 10(a), 10(b) and 11 show the block diagram, schematic, and PCB layout of the microcontroller board. The microcontroller unit packages are illustrated is illustrated in Figs. 12 and 13.

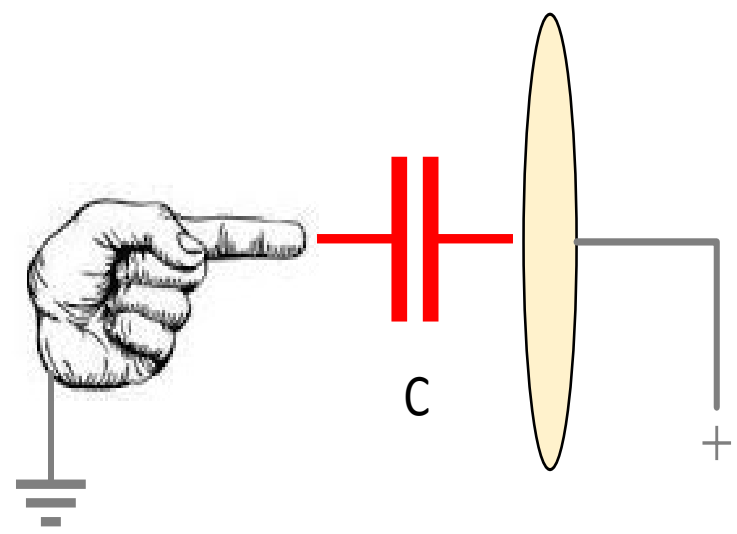

Fig. 6: Interaction of the human body with the capacitive electrode

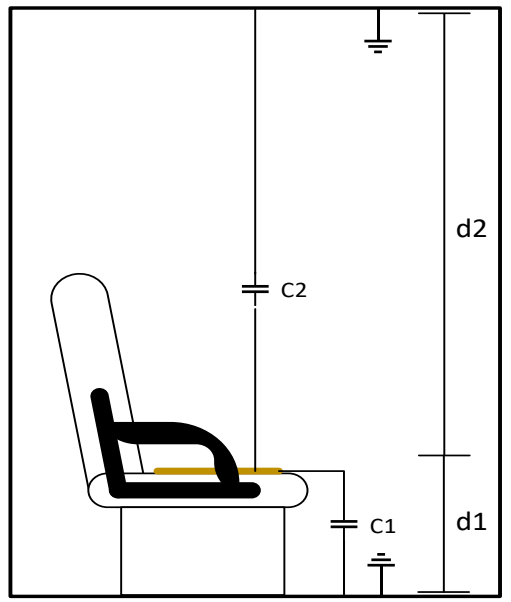

Fig. 7: A model of capacitances between sensor electrode and auditorium building 


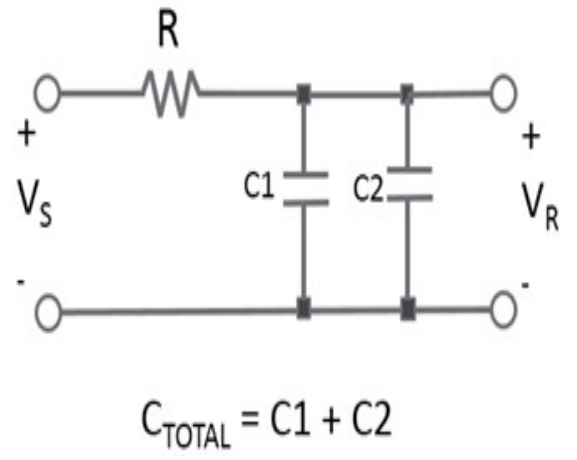

Fig. 8: Circuit representation of the capacitive sensor

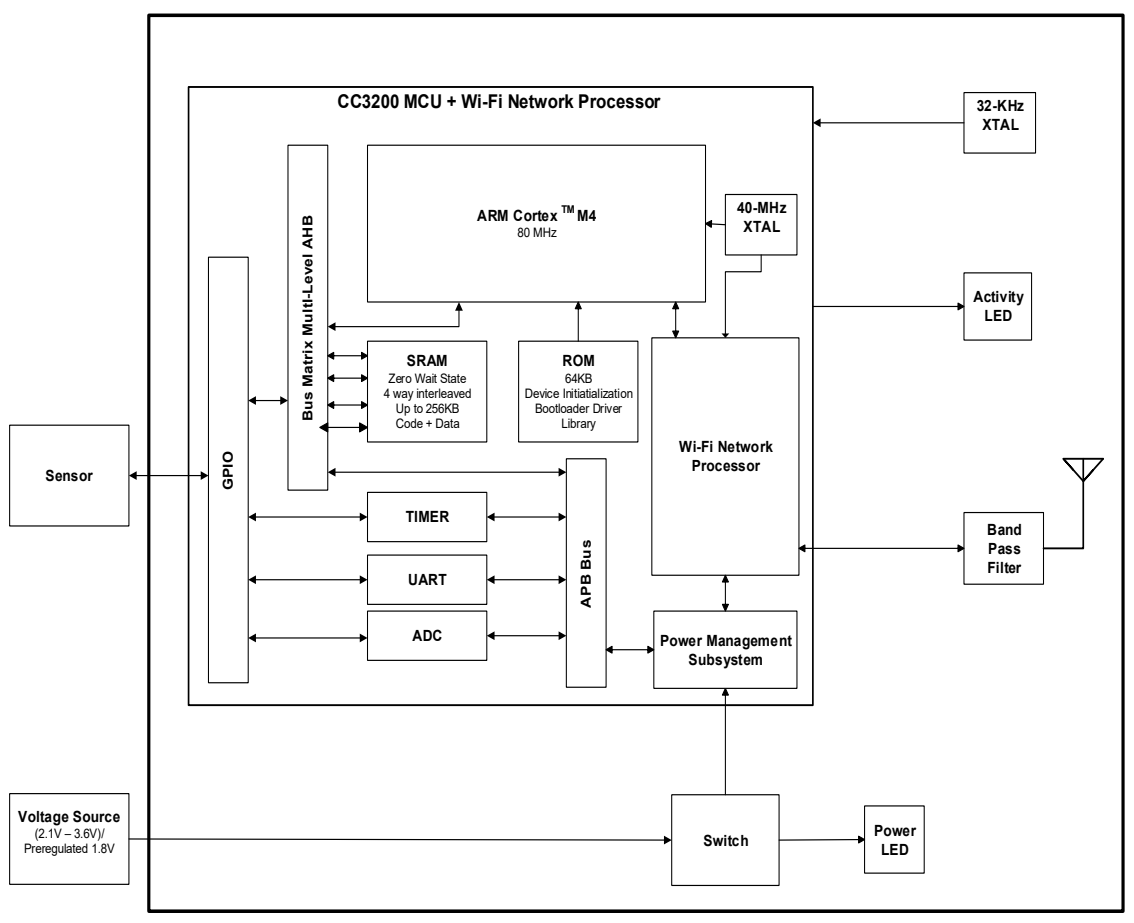

Fig. 9: Block diagram of microcontroller unit with attached sensor 
Design and implementation of seat occupancy detection system... 34

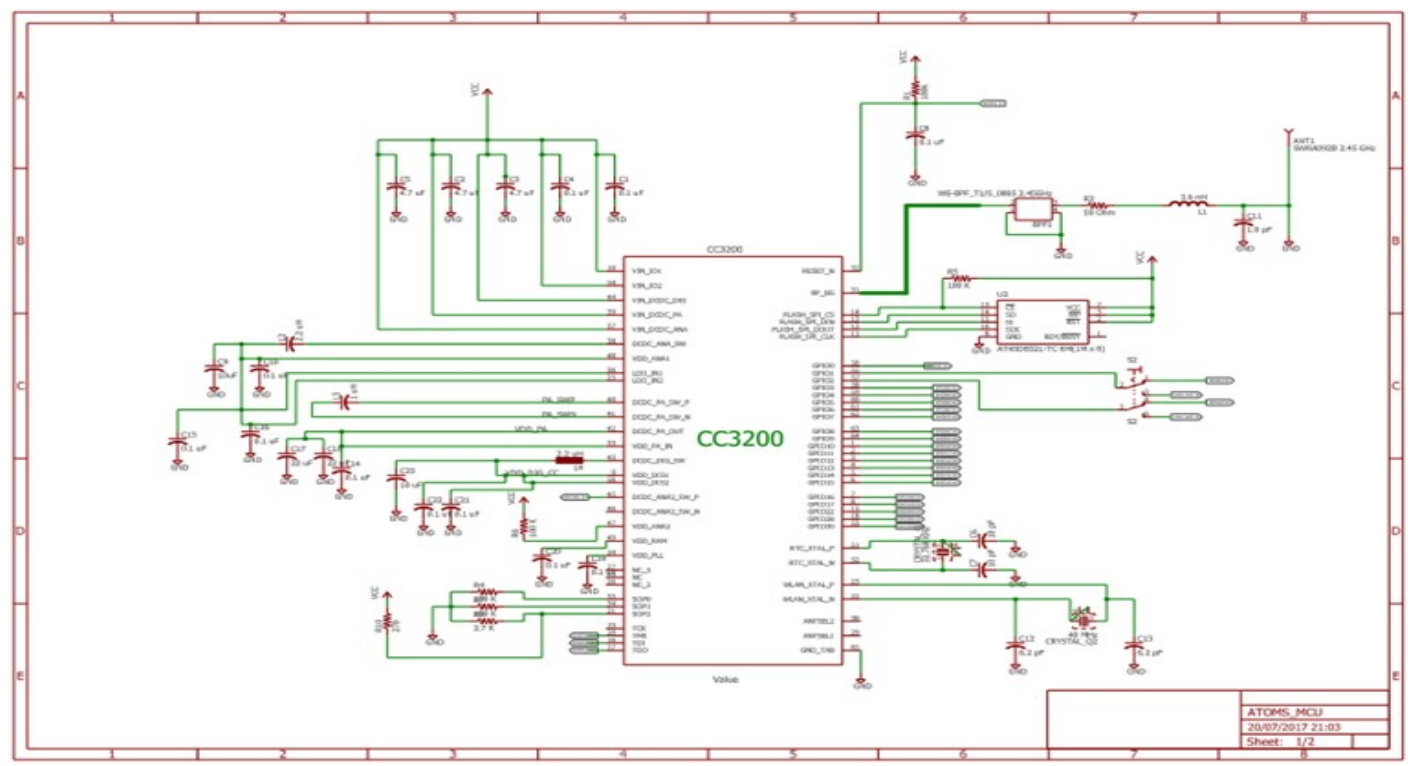

Fig. 10(a): Schematic of the microcontroller board showing the TI CC3200 microcontroller and antenna connections

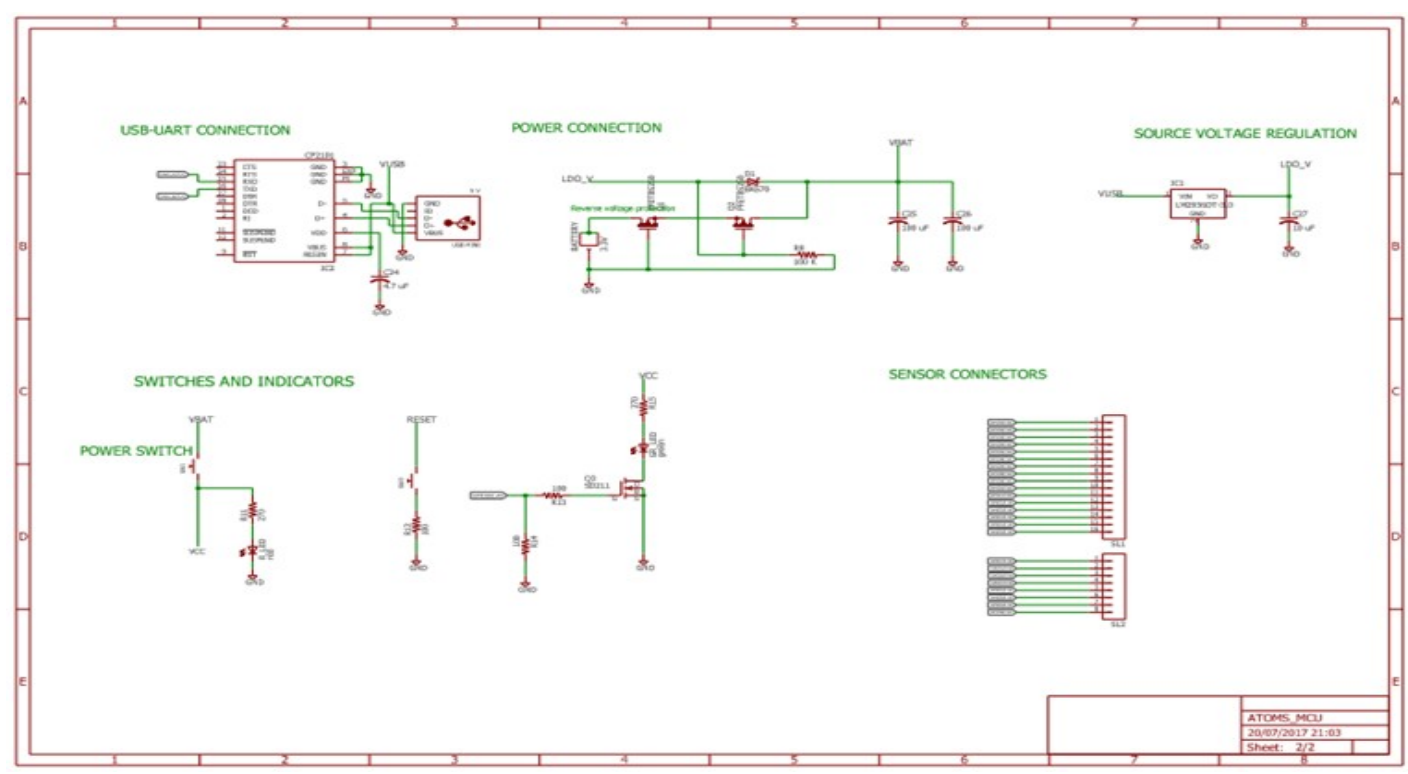

Fig. 10(b): Schematic of the microcontroller board showing switches, indicators, power, and programming interface connections 


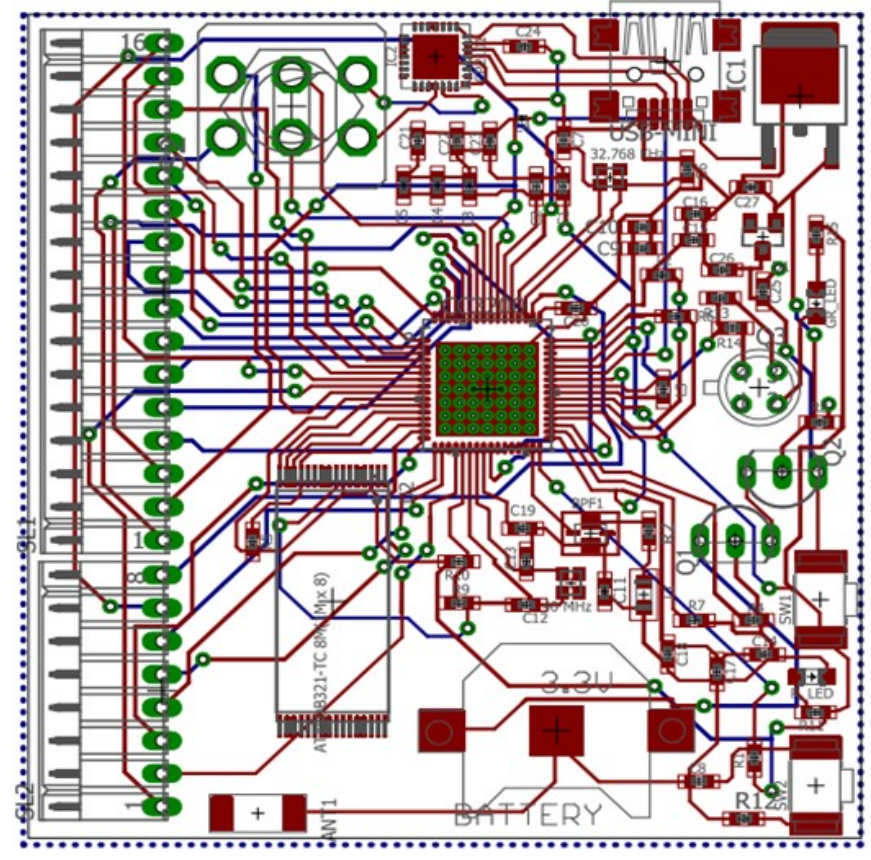

Fig. 11: PCB layout of microcontroller board

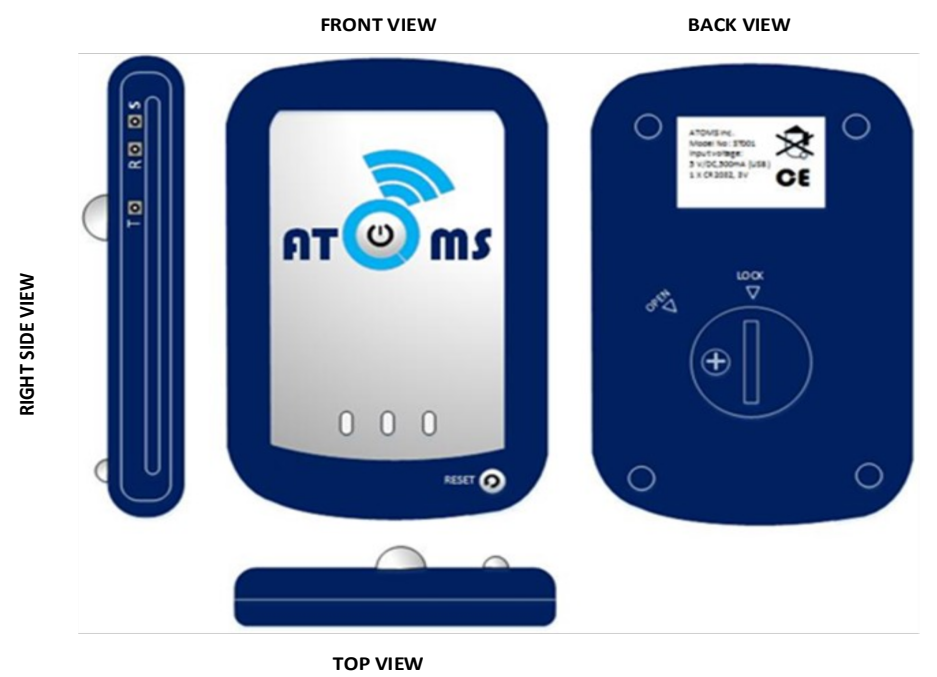

Fig. 12: Sensor-cluster microcontroller device packaging 

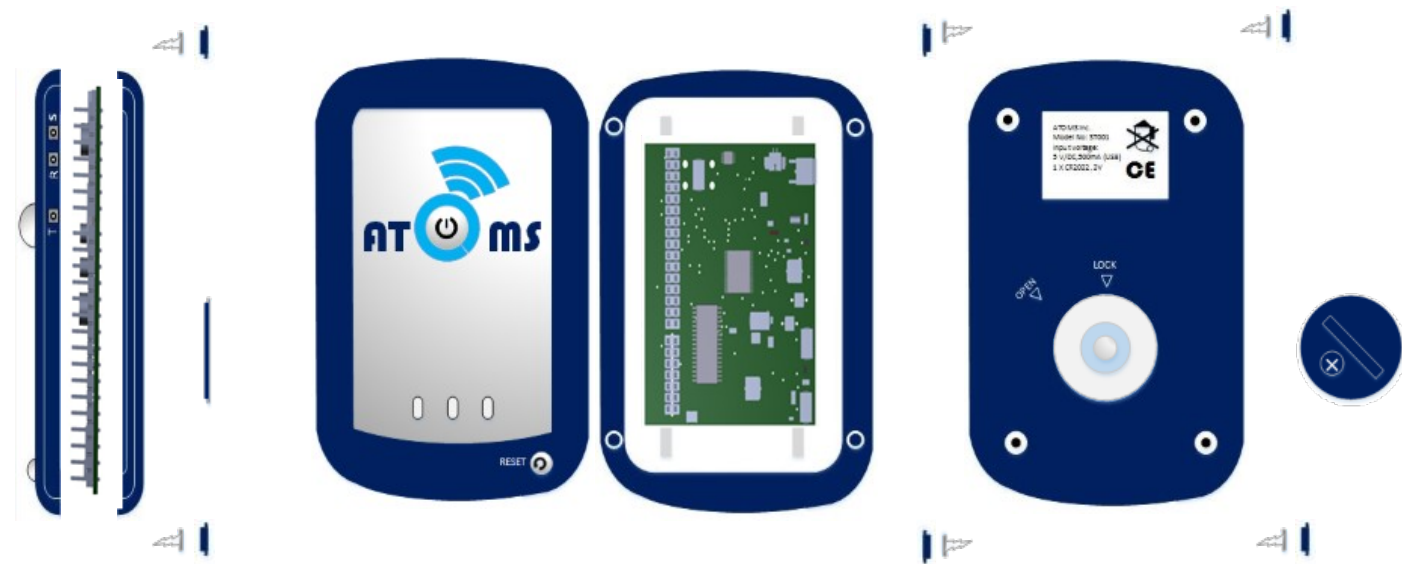

Fig.13: Exploded view of sensor-cluster microcontroller device

\section{Base Station}

A laptop computer with Wi-Fi capability was used as the base station because of its portability. The base station had a software application named ATOMS (Auditorium and Theatre seat Occupancy Monitoring System) which run on a local server. This software received occupancy data from the microcontroller and served as a visualization platform for the processed information. As shown in fig 14, the graphical representation of the processed information could also be projected onto a larger display device at vantage points for use by patrons. Additionally, control signals could be sent wirelessly to the cluster devices from the base station.

The software required that a base station administrator logged in with their credentials to access the system via their account. Since the software is a multi-usersystem, a successful login into an administrator's account gives a user authorization to access certain resources provided by the system based on the access level. This was done using identity correlation for the purposes accountability, security, logging and resource management.
The startup view of the main page of the base station application is shown in Fig.14. The left bar houses HALL VIEW, OPTIONS, and ALLOCATE menu buttons which are involved in the major actor-system interactions. The lower section of the bar gives numerical information concerning the total available seats in the hall. The section of the page right of the menu bar shows a graphical and numerical representation of seats in the auditorium. Below each column of seats is the number of available seats in that column. On the software's interface, seats are represented by colour filled circles. The color that a circle bears at any instant indicates the occupancy or physical status of the corresponding seat. The colour scheme employs three colors: light cadet blue, reddish orange and black which signal vacancy, occupancy, and state of seat or sensor unit respectively.

The HALL VIEW button which is the normally active button in the menu bar upon login, closes all extended panels and reverts to the post-login view when clicked as shown in Fig. 14. The menu also has an OPTIONS button which when clicked, extends a panel to the right of the bar. As shown Fig. 15, the panel has a logout button and subpanels for tagging seats as faulty, 


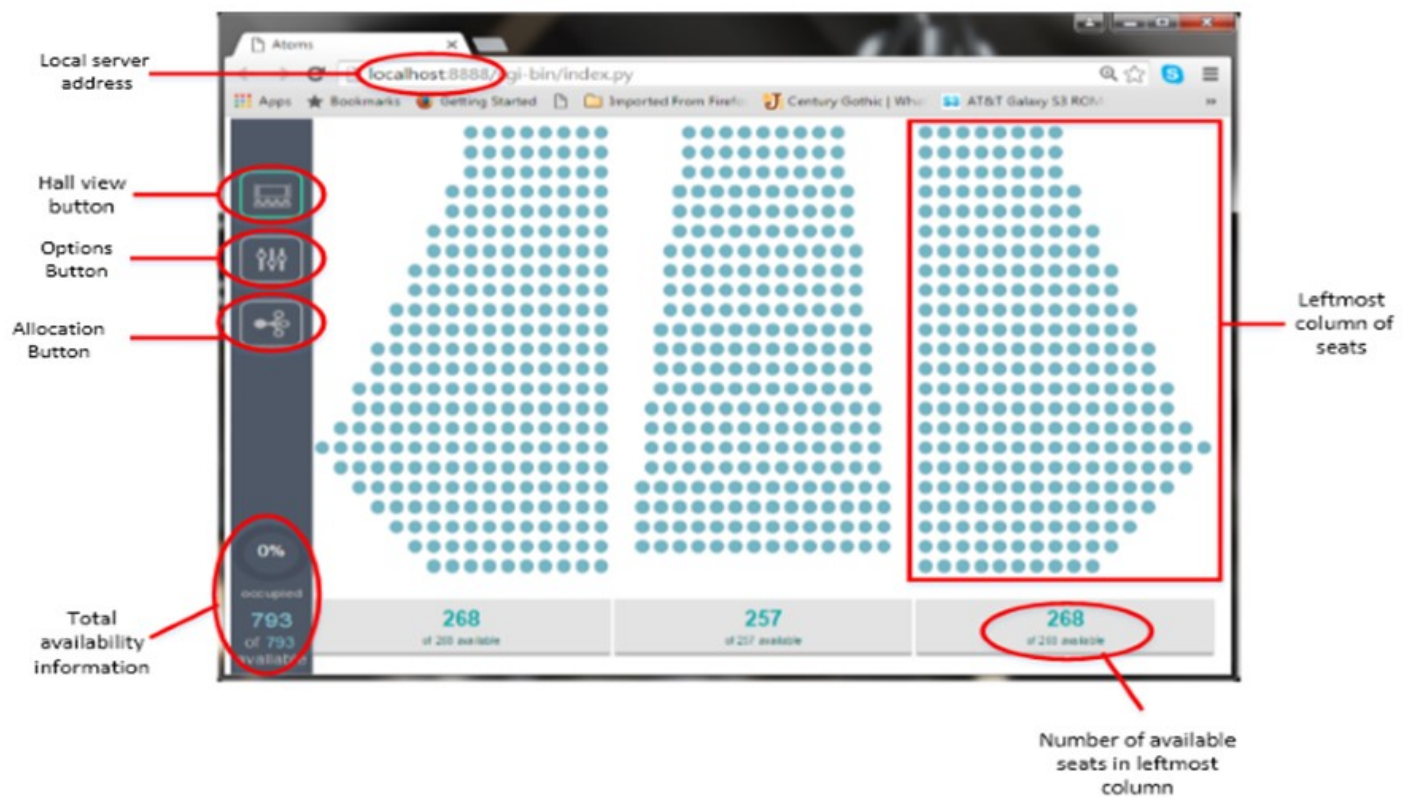

Fig. 14: The base station software showing the hall view on startup

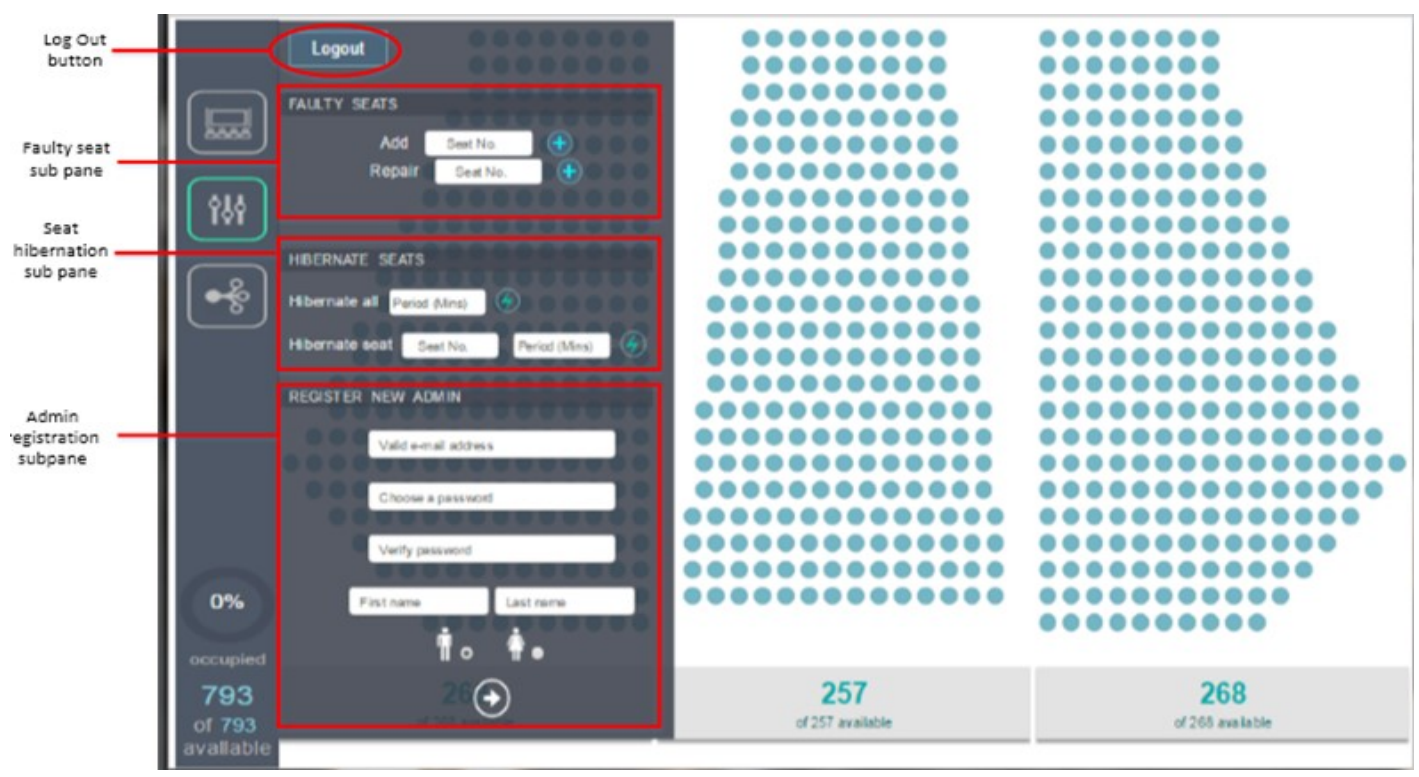

Fig. 15: A view of the OPTIONS panel 
manually putting seat cluster devices in deep sleep modes, and creating accounts for new administrator. Clicking the ALLOCATION button opens the ALLOCATION pane as shown in Fig. 16 where the administrator is given the controls needed to reserve seats for some clients or otherwise. Additionally, the panel provides lists of vacant and reserved seats in the auditorium. Fig. 19 is a view of the application interface showing hall seats and their statuses.

\section{EXPERIMENTAL SETUP AND RESULTS}

A prototype of the seat occupancy detection system consisting of the sensor electrode, $\mathrm{WiFi}$ -enabled microcontroller and the base station device was setup in the hall seating area. The prototype was subjected to testing in a typical hall environment in order to achieve precise simulation of the real-world setting. Fig. 17 shows the prototype's test setup.

Ten tests were run on the capacitive sensor to determine its performance. In these tests, the charge and discharge times of the sensor were measured from charge-discharge plots obtained from an oscilloscope. For plot with $V_{c c}=3.0 \mathrm{~V}$ and $V_{H T}$ and $V_{L T}$ measured as $2.5 \mathrm{~V}$ and $1.5 \mathrm{~V}$ the results of the seat sensor tests are summarized in Table 1. The charge-discharge plot from the first test of the sensor is shown in Fig. 18 .

The average charge and discharge times for the unoccupied seat states were calculated As

$t_{C(\text { UNOCP })}=0.560 \mathrm{~ms}$ and $t_{D(\text { UNOCP })}$

$=0.214 \mathrm{~ms}$ respectively. The average occu-

pied state charge and discharge times were also $t_{C(O C P)}=1.416 \mathrm{~ms}$ and $t_{D(O C P)}=0.374 \mathrm{~ms}$ respectively. With $V_{S}=3.0 \mathrm{~V}, V_{R}$ (CHARGE) $=2.5 \mathrm{~V}$, $V_{R}$ (DISCHARGE) $=1.5 \mathrm{~V}$, and $R=50 \mathrm{M} \Omega$, the capacitance was determined from

$C=-\frac{t}{R \ln \left(1-\frac{V_{R}}{V_{S}}\right)}$

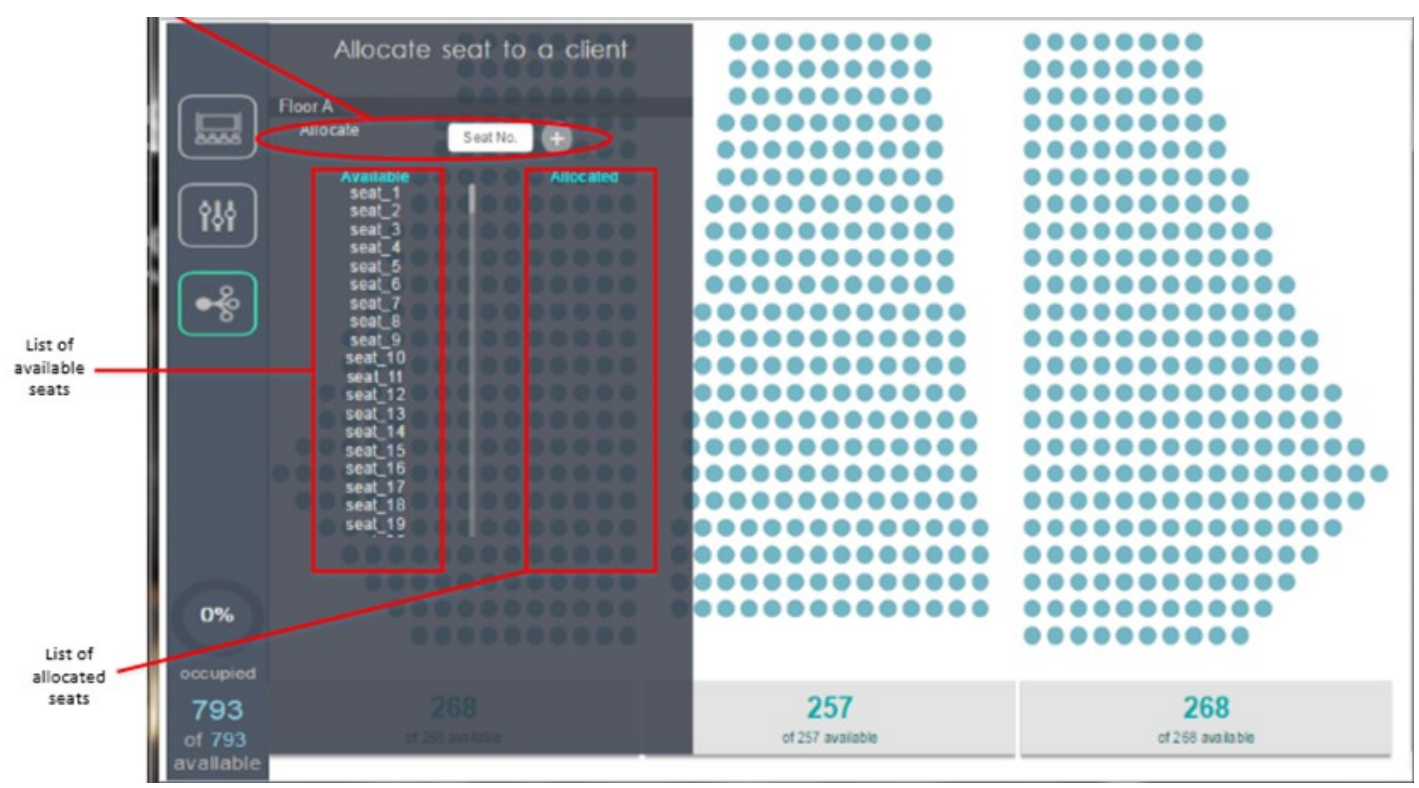

Fig. 16: A view of the ALLOCATION panel 


\section{Kommey et al.}

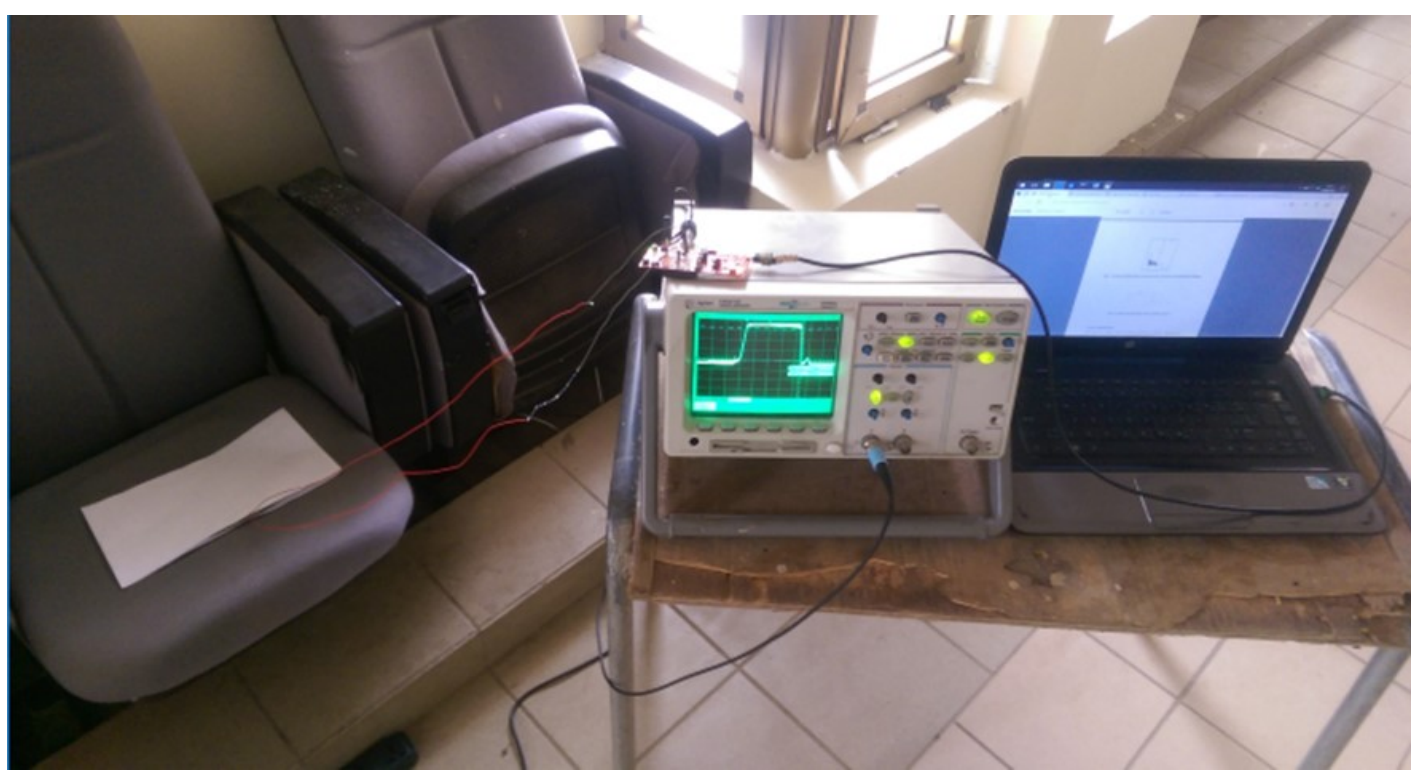

Fig. 17: Test setup of prototype

Table 1: Charge and discharge times of sensor electrodes for each test

\begin{tabular}{cccc}
\hline Test & Seat state & Charge time $(\boldsymbol{m s})$ & Discharge time $(\boldsymbol{m s})$ \\
\hline 1 & Unoccupied & 0.52 & 0.22 \\
2 & Occupied & 1.43 & 0.41 \\
3 & Unoccupied & 0.55 & 0.20 \\
4 & Occupied & 1.40 & 0.30 \\
5 & Occupied & 1.45 & 0.36 \\
6 & Occupied & 1.39 & 0.42 \\
7 & Unoccupied & 0.58 & 0.20 \\
8 & Unoccupied & 0.57 & 0.22 \\
9 & Occupied & 1.41 & 0.38 \\
10 & Unoccupied & 0.58 & 0.23 \\
\hline
\end{tabular}

The average unoccupied seat state charge and discharge capacitances were calculated from equation (6) as $C_{C \text { (UNOCP) }}=6.250 \mathrm{pF}$ and
$C_{D(\text { UNOCP })}=6.174 \mathrm{pF}$ respectively resulting in a mean unoccupied state capacitance of $C$ $($ UNOCP $)=6.212 p F$. Occupied seat state charge 
and discharge capacitances were also evaluated from Equation (6) as $C_{C(O C P)}=15.805 p F$ and $C_{D(O C P)}=10.79 p F \quad$ respectively. This resulted in a mean occupied state capacitance of $C_{(O C P)}$ $=13.2975 \mathrm{pF}$. Empirical capacitance values were quite close to the theoretically calculated values with $2.899 \%$ and $2.782 \%$ differences for unoccupied and occupied states respectively. These discrepancies are as a result of the approximations made in theoretical calculations and also electrical interactions between the sensor and the metallic structures in the seat. The relationship between the occupied unoccupied seat state capacitances was established as $C_{(O C P)} \approx{ }^{2 \mathrm{C}}{ }_{(U N O C P)}$. Since the occupied seat state capacitance was more than double the unoccupied seat state capacitance, it is proven that the occupancy detection system can clearly differentiate seat states. The system is therefore a viable option for the detection of human occupants on seats. An additional three-day integrated system test was conducted which involved random occupancy, vacation, allocation, de-allocation, singular and mass hibernation, fault seat tag assignment and removal. Several arbitrary log in and log out instances by various administrators; and registering of new administrators also took place. The power consumption of the device was also monitored. The local area network was also monitored using the PTRG Network Monitor ${ }^{\circledR}$. After the three day test, the dc power supply had recorded a $6 \%$ voltage drop from an initial $3.0 \mathrm{~V}$. The desktop application and seat unit communication however experienced four percent packet failure as indicated by the network monitor. This was attributed to factors such as electromagnetic interference and radio range issues.

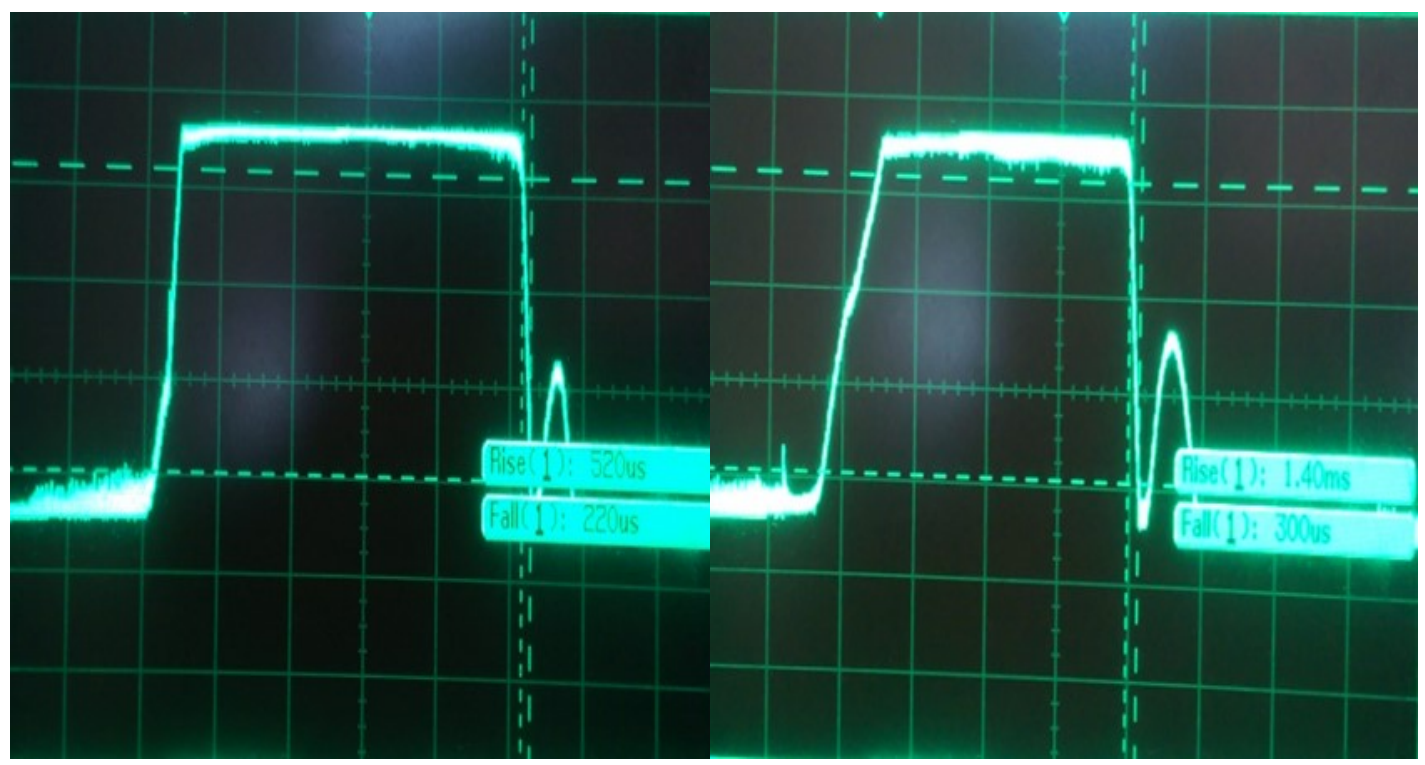

(a)

(b)

Fig. 18: Charge-discharge plot from Test 1 of sensor showing charge and discharge times of unoccupied (a) and occupied (b) seat states 


\section{Kommey et al.}

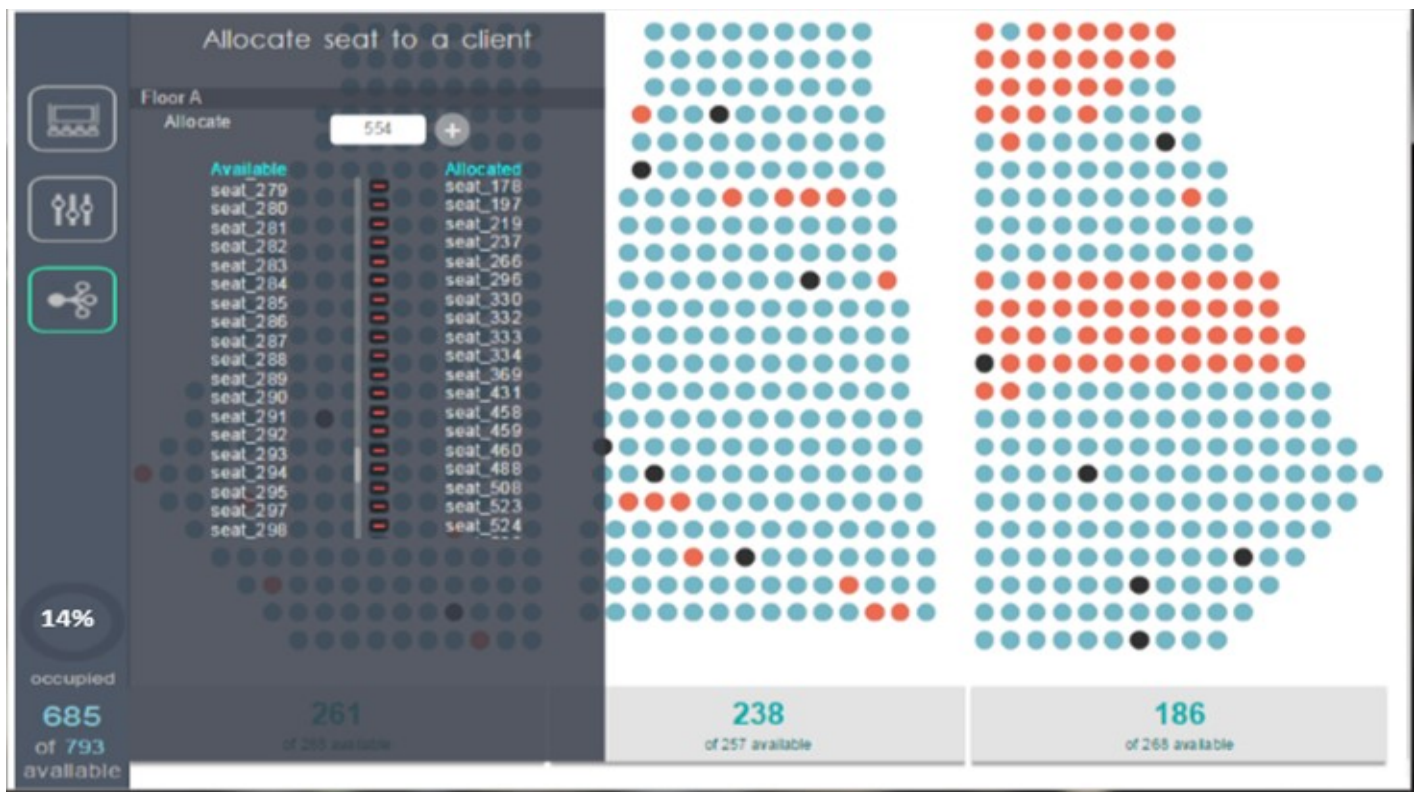

Fig. 19: A view of the software interface showing seats with their respective status

\section{CONCLUSION}

We have proposed and tested a system for dynamically monitoring seat occupancy in auditoriums, halls and the like. From empirical and theoretical results, the occupied seat state capacitance was more than double the unoccupied seat state capacitance. This proves that the occupancy detection system clearly differentiates between the two seat states. The system is therefore a viable option for the detection of human occupants on seats. Compared to other occupancy detection and monitoring systems, the system is more efficient, accurate and cost effective. Wireless communication adapted into this system reduces cabling require for communication and control making seat occupancy monitoring easier and cheaper. The designed system was highly practical and adaptable for installation in any auditorium setting. The system is also very portable and can be used to cater for already built seats. It also enables hall establishments to operate independent of ushers.

\section{REFERENCES}

Bahl, I. J. and Triveda, D. K. (1997). A designer's guide to microstrip line. Microwaves, $16(5): 174-176$.

Baron, H. E. and Abbott, R. M. (2000). Seat occupancy detector and seating area display system. United States Patent : US 6,140,921: $1-16$.

Blinkilde, P. J.and Sandi, F. J. (1975). Occupant sensor seat switch. United States $\mathrm{Pa}$ tent : US 3,859,485: 1-8.

Gabriel, C., Gabriel , S. and Corthout, E. (1996). The dielectric properties of biological tissues: I. Literature survey. Physics in Medicine and Biology (41): 2231-2249.

Gaugler, D. D. and Gaugler, E. E. (1941). 
Chair for motion picture theaters. United States Patent : US 2,257,496: 1-4.

George, B., Zangl, H., Bretterklieber, T. and Brasseu, G. (2009). Seat occupancy detection based on capacitive sensing. Instrumentation and Measurement, IEEE Transactions on, 58 (5): $1487-1494$.

Grunfeld, P. (2012). Seat occupancy detection and display system. United States Patent: US 2012/0242492 A1, 1-11.

Hefferon, M. T. (2013). Dynamic Occupancy Monitoring. United States Patent : US 8,587,446 B2: 1-14.

Helbling, E. and Glass, A. (1998). Automatic theater ticket concierge. United States $\mathrm{Pa}$ tent : US 5,797,126: 1-12.

Hoch, E. (1926). Electrode effects in the measurement of power factor and dielectric constant of sheet insulating materials. Bell System Technical Journal, 5: 555.

Satz, A., Hammerschmidt, D. and Tumpold, D. (2009). Capacitive passenger detection utilizing dielectric dispersion in human tissues. Sensors and Actuators A: Physical, 152, (1): $1-4$.

Schroeder, M. J., Sasasiva, A. and Nelson, R. M. (2008). Journal of Biomechanics, Bio- medical and Biophysical Engineering, 2 (1): $1-11$.

vonDoenhoff, R. C. and Shetty, S. S. (2008). Automatic airplane seat location mapping. United States Patent : US 7,319,854 B2: 1-8.

Watson, P., Watson, I.and Batt, R. (1980). Total body water volumes for adult males and females estimated from simple anthropometric measurements. The American Journal of Clinical Nutrition, 33(1): 27-39.

Wimmer, R. (2011). Capacitive sensors for whole body interaction. Human-Computer Interaction Series , 121-133.

Zangl, H., Bretterklieber, T., Hammerschmidt, D. and Werth, T. (2008). Seat occupancy detection using capacitive sensing technology. SAE Technical Paper 2008-01-0908, 110.

Zeeman, A. S., Booysen, M. J., Ruggeri, G. and Lagana', B. (2013). Capacitive seat sensors for multiple occupancy detection using a low -cost setup. International Conference on Industrial Technology (pp. 1228-1234). IEEE.

Zeidler, F., Petri, V., Mickeler, R. and Meyer, M. (1997). Device for detecting seat occupancy in a motor vehicle. United States $\mathrm{Pa}$ tent : US 5,612,876: 1-7. 